\title{
Medusahead Management in Sagebrush-Steppe Rangelands: Prevention, Control, and Revegetation
}

\author{
By Dustin D. Johnson and Kirk W. Davies
}

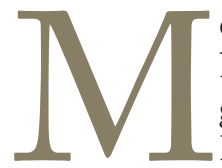

edusahead (Taeniatherum caput-medusae [L.] Nevski) (Fig. 1) is an aggressive exotic annual grass native to the Mediterranean region of Eurasia that is changing the ecology and productivity of western rangelands. Since the collection of the first known North American herbarium specimen of medusahead in 1887 near Roseburg, Oregon, ${ }^{1}$ the exotic annual grass has invaded millions of acres in the Pacific Northwest, California, Utah, Nevada, and Colorado. On public lands administered by the Bureau of Land Management, 3.3 million acres of rangeland are classified as monotypic stands of cheatgrass (Bromus tectorum L.) and/or medusahead, nearly 14 million acres are infested with one or both grasses, and over 62 million acres are at risk of invasion by the exotic annual grasses. ${ }^{2}$

Medusahead invasion is a serious management concern because it reduces harvestable forage for domestic livestock and wildlife, decreases biodiversity, and increases the frequency and size of wildfires. Much of medusahead's success as an invader can be attributed to its ability to greatly limit seedling establishment of desirable perennial plant species through competition, ${ }^{3,4}$ suppression, ${ }^{5}$ and alteration of fire cycles. ${ }^{5-7}$ Medusahead is extremely competitive, especially on heavy, clayey soils, and has even displaced cheatgrass in areas of Idaho. ${ }^{8}$ It often germinates in the fall and retains the ability for root growth at lower temperatures during winter and early spring and is able to capitalize on and deplete soil moisture when most desirable grasses are in a dormant state. ${ }^{4}$ Invasion by this exotic annual grass frequently results in the formation of a dense layer of litter that decomposes slower than other plants because of a high silica content. ${ }^{5}$ The resulting highly persistent thatch layer suppresses native plant growth, while promoting the germination of medusahead seed. ${ }^{5}$ An accumulation of medusahead litter also increases the amount and continuity of fine fuel, which can increase the frequency of wildfires to the detriment of native vegetation. ${ }^{5-7}$ More frequent fire not only escalates risk of mortality for desirable vegetation, but also produces disturbed land that is easily infested with more medusahead. Medusahead, like cheatgrass, is favored by conditions of higher resource availability associated with a disturbance event such as wildfire. Similar to other exotic annual grasses, revegetation of medusahead-invaded plant communities is expensive and often unsuccessful because seeded desirable vegetation rarely establishes.,9 In addition, invasion by this exotic annual grass can greatly reduce the grazing capacity of rangelands, ${ }^{7}$ making it clear that medusahead can cause significant economic losses for landowners and ranchers.

Given the magnitude of negative impacts and the alarming rate of increase associated with medusahead invasion on western rangelands, there is a critical need among landowners and managers for management options for areas invaded by or at risk of being invaded by medusahead. In this paper, we will present the best management options based on what is currently known about medusahead prevention, control, and revegetation on sagebrush-steppe rangelands.

\section{Medusahead Management Options}

Management options for medusahead, and any other invasive plant for that matter, fall under the primary categories of prevention, control, and revegetation. A successful approach to managing medusahead within a particular management area or region likely includes strategies that fall under all three categories. A medusahead management area can be defined in many ways, but for the purposes of this paper we will define it as a particular geographic region in which all landowners and managers have a common interest in controlling existing populations and the future spread of medusahead.

\section{Options for Limiting the Spread of Medusahead}

In many cases, medusahead occupies a relatively small proportion of its potential range in any given management area. In these situations, a primary objective for managing medusahead is controlling its future spread within the management area. The following three principal strategies 


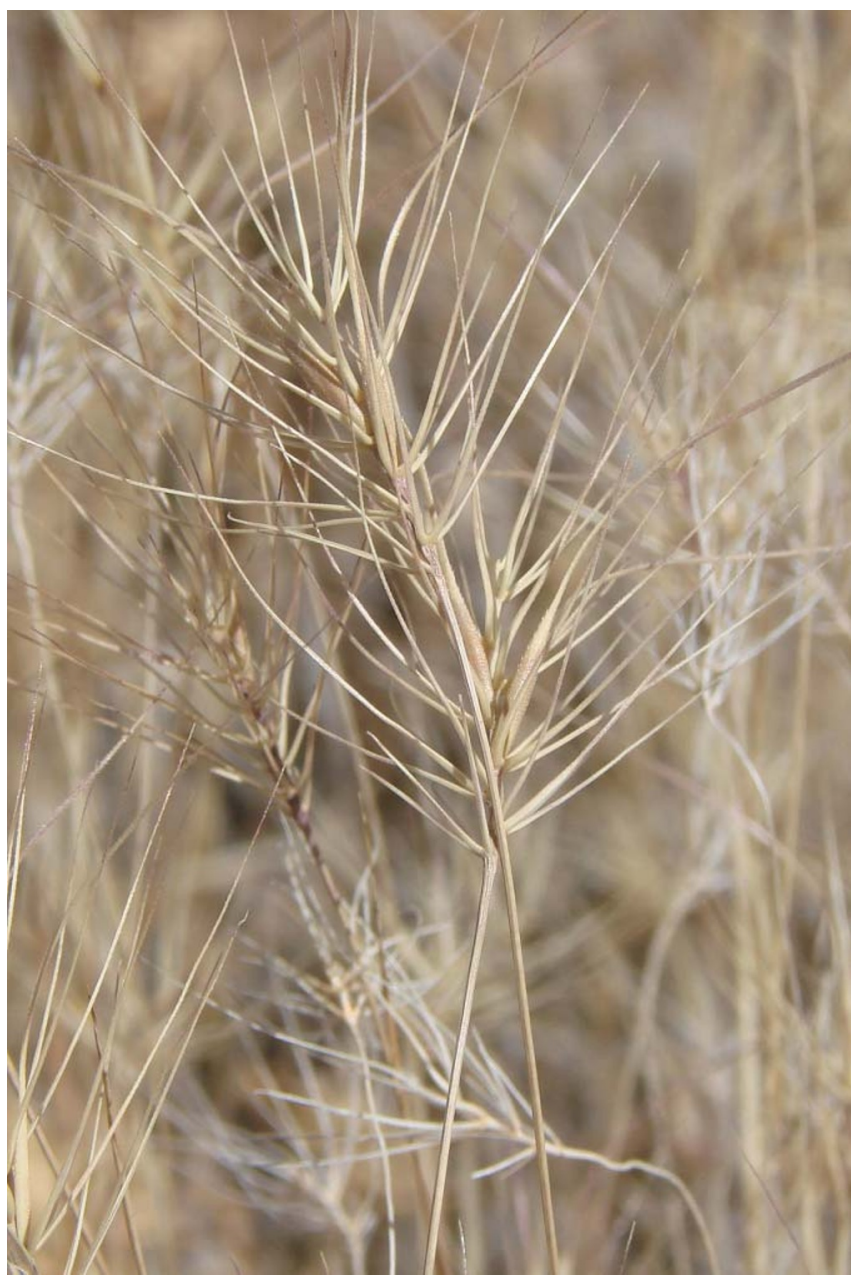

Figure 1. Photo showing a macro view of medusahead inflorescences.

should be used for preventing the continued spread of medusahead: 1) limiting medusahead seed from dispersing to noninvaded area, 2) reducing resource/safe site availability to medusahead, and 3) eradicating new medusahead infestations. ${ }^{10}$

\section{Limiting Spread of Medusahead Seed}

The vast majority of medusahead seeds disperse relatively short distances $(<2 \mathrm{~m})$ from the source population (Fig. 2), ${ }^{11}$ suggesting that containment zones around medusahead infestations need only be a few meters wide to effectively suppress invasion into surrounding plant communities. In a study in southeast Oregon, Davies et al. ${ }^{12}$ found that a $6-\mathrm{m}$ wide barrier of desert wheatgrass (Agropyron desertorum [Fisch. ex Link] Schult.), established directly adjacent to infestation edge, resulted in a 40 -fold decrease in the spread of medusahead over a 2-year period (Fig. 3). In other words, the density of medusahead beyond the vegetation barrier of desert wheatgrass was 40 -fold less than beyond where a vegetation barrier had not been established. However, some medusahead seed dispersed and established beyond the desert wheatgrass barrier in the study. The authors suggested

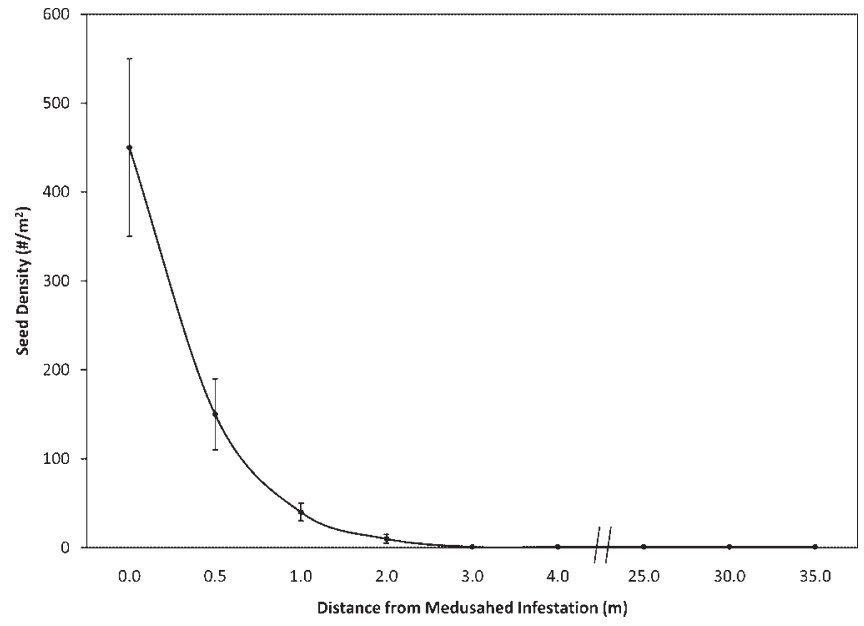

Figure 2. Medusahead seed density (mean \pm SE) at varying distances from invading front of medusahead infestations (Adapted from Davies $\left.{ }^{11}\right)$.

the effectiveness of vegetation barriers might be improved by making them wider and incorporating an early detection and eradication program for satellite populations that establish beyond the vegetation barrier. In addition, the authors suggested the vegetation barrier might have been more effective if had been established further from the infestation edge. This would have allowed the competitive vegetation to become better established prior to experiencing competition from the invader. The competitive vegetation barrier of desert wheatgrass, a deep-rooted perennial bunchgrass, reduced the spread of medusahead in the study by reducing the spatial dispersal of seeds through physical interception, decreasing the availability of resources for establishment of the invader, and increasing the distance seeds needed to

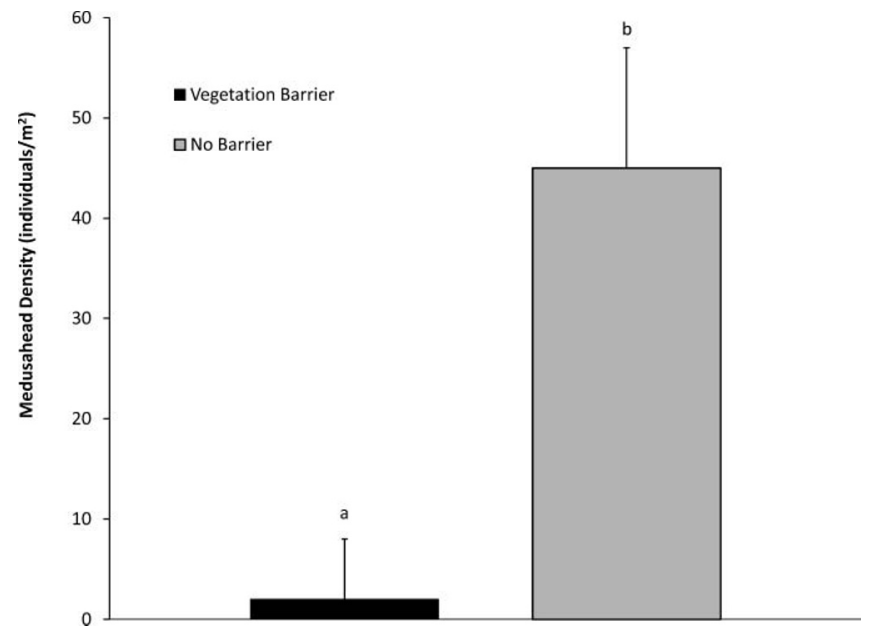

Figure 3. Medusahead densities in plant communities protected by a desert wheatgrass barrier and plant communities not protected by a barrier (mean $\pm \mathrm{SE}$ ). Different lowercase letters indicates a difference in medusahead density between treatments $(P \leq 0.05$; Adapted from Davies et al. ${ }^{12}$ ). 
disperse to find suitable conditions for establishment. In areas where complete control of medusahead cannot be obtained (i.e., large, persistent infestations) or where use of effective controls is precluded by terrain or policy, focusing on establishment of a competitive buffer of deep-rooted perennial grasses around the perimeter or along the invading front of medusahead populations would be appropriate to greatly reduce the spread of medusahead. The economic costs associated with ensuring the establishment of such a barrier are justified by the number of acres protected from invasion by medusahead within a given management area.

Although representing a minority of seeds produced by medusahead, seeds that are dispersed long distances are the most troublesome for land managers because they have the potential to establish new infestations in new locations within a management area. The key to reducing longdistance dispersal of medusahead seed is to focus on limiting their contact with vectors, such as vehicles and animals, during the period of seed disarticulation. We suggest that it would be appropriate to focus a large proportion of management resources toward controlling medusahead along roads and trails to prevent seed production and eventual dispersal by vehicles and animals. Again, we contend a disproportionate use of limited management resources would be justified by the number of acres protected from new introductions of medusahead. Unlike vehicles, animal movements are not limited to established routes; therefore, it would be difficult to focus control efforts on particular vector pathways to prevent contact of animals with medusahead during seed disarticulation. Despite there being few options to control the location and timing of wildlife and feral animal use of areas infested with medusahead, the timing and location of domestic livestock grazing can be controlled. Careful consideration should be given to the order and timing of pasture use in management areas containing medusahead. Davies ${ }^{11}$ suggested that livestock should probably be prevented from travelling from medusahead infestations to noninvaded plant communities during the months (July to October), when seeds disarticulate, in order to limit dispersal of medusahead. This strategy would be especially critical in pastures that have a relatively small proportion of their total acreage occupied by medusahead, and might be less critical in pastures that are more fully occupied by medusahead; careful consideration should be given to order of pasture use in either situation to prevent spread from one pasture to the next. More research is needed on livestock behavior in medusahead-infested areas to better inform grazing management decisions for reducing the spread of medusahead.

\section{Reducing Resource Availability to Medusahead}

Plant communities are predicted to become more susceptible to invasion whenever there is an increase in the amount of unused resources. ${ }^{13}$ A primary objective for limiting the spread of medusahead should be to reduce the amount of unused resources available on which medusahead can capitalize in sagebrush plant communities. The only practical way to reduce resource availability to medusahead extensively established on rangeland is to promote/maintain a competitive plant community. In most cases this will require establishing or maintaining a vigorous perennial bunchgrass population on sagebrush-steppe rangelands. There is a strong negative association between perennial bunchgrass and medusahead, whereby medusahead density decreases as perennial bunchgrass density increases. ${ }^{11}$ The negative association provides evidence that established perennial bunchgrasses play a central role in reducing resource availability to medusahead. This ability to limit resource availability to medusahead is perhaps most-related to their superior ability to sequester resources, coupled with a large overlap that exists in the temporal and spatial acquisition of resources between medusahead and perennial bunchgrasses. ${ }^{14}$ Although prolonged drought or other climatic anomalies can significantly affect perennial bunchgrass density, factors influenced by management that have great potential to impact perennial bunchgrass populations on rangeland include livestock grazing, fire, and their interaction. Proper grazing management has little or no negative impact on perennial bunchgrasses. However, improper or continuous heavy livestock grazing has been shown to compromise the competitive ability of perennial bunchgrasses and decrease density, in some cases. Proper grazing management that incorporates periodic growing season rest for perennial bunchgrasses is critical for maintaining bunchgrass density and thereby reducing resource availability to medusahead. For example, in a study of the influence of crested wheatgrass defoliation intensity and season on medusahead invasion, Sheley et al. ${ }^{15}$ concluded that on clayey and loamy soils, established crested wheatgrass is capable of resisting invasion by medusahead if plants are allowed to fully recover and regain their biomass production from one grazing season to the next.

Wildfire is another factor that can negatively impact perennial bunchgrass populations. Fire can cause substantial mortality of perennial bunchgrasses. In addition, medusahead is well-adapted to establish in recently burned areas. The loss of established perennial bunchgrasses to fire mortality coupled with conditions favoring exotic annual grasses, often leads to medusahead dominance following fire. There are three primary strategies for reducing the impacts of wildfire to perennial bunchgrasses and subsequent exotic annual grass invasion. One is to prevent wildfires from occurring. This strategy has proven to be untenable, because most areas of sagebrush-steppe rangeland will eventually burn despite fire suppression and prevention efforts. Removing the role of fire has also led to unintended ecological consequences in some sagebrush ecosystems. For example, a reduced role of fire in higher elevation sagebrush rangelands has allowed for a large-scale increase in woody species (e.g., western juniper, piñon pine); representing an undesirable shift in vegetation replete with its own host of negative ecological and economic impacts. A different strategy employs postfire seedings of 


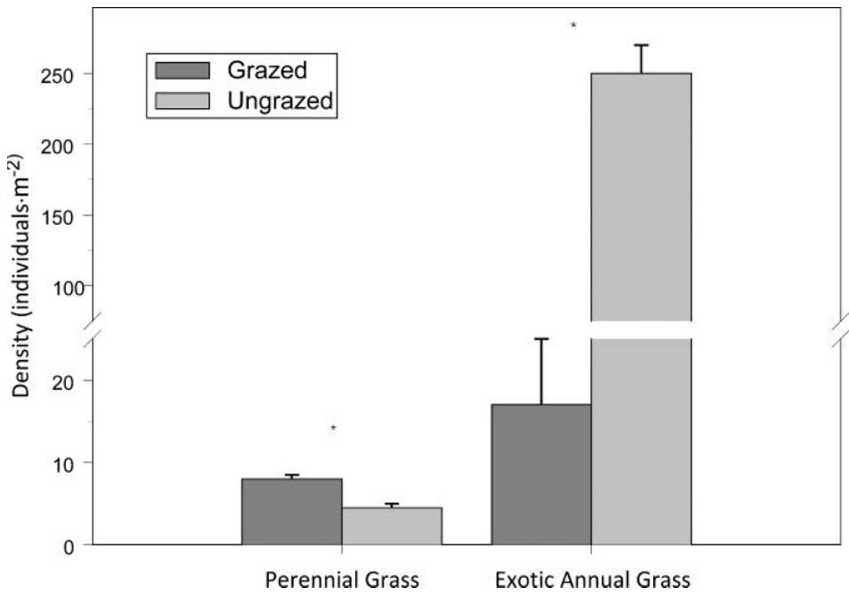

Figure 4. Density of perennial bunchgrasses and exotic annual grasses averaged over 12, 13, and 14 years after fire. Treatment key: Grazed $=$ moderately grazed by livestock from 1936 until 2 years prior to the burn and Ungrazed $=$ livestock excluded since 1936. Asterisk $\left(^{*}\right)$ indicates significant difference $(P \leq 0.05)$ in plant density between treatments (Adapted from Davies et al. ${ }^{17}$ ).

competitive perennial bunchgrasses. Land managers often must resort to this approach despite it being expensive and frequently unsuccessful. In addition, seeded grasses are initially much less effective for reducing resource availability to medusahead and other exotic annual grasses than established perennial bunchgrasses; seedlings simply uptake resources less readily than established, mature plants. ${ }^{16}$ The most efficient and perhaps most effective strategy is to manage existing perennial bunchgrass populations in a way that best prepares them to tolerate and survive a wildfire. In a southeast Oregon study, Davies et al. ${ }^{17}$ found that longterm livestock grazing exclusion increased risk of exotic annual grass invasion following a fall burn compared to areas that had been moderately grazed over the same time period. The authors defined moderate livestock grazing as approximately $40 \%$ annual use that incorporated periodic growing season rest for perennial bunchgrasses. The increase in exotic annual grass density in areas that had been protected from livestock grazing persisted for 14 years following fire (Fig. 4). The authors suggested that moderate levels of livestock grazing reduced litter accumulation of perennial bunchgrasses that resulted in more vigorous plants that experienced less self-shading and reduced fuel loading atop grass crowns (Fig. 5). A reduced amount of fuel loading on the crowns of plants that had been moderately grazed afforded perennial bunchgrasses better survival following fire. Greater survival and greater density of mature perennial bunchgrasses in grazed areas following fire greatly reduced postfire resource availability to exotic annual grasses compared to areas that had been afforded long-term protection from livestock grazing and experienced high perennial bunchgrass mortality in response to the burn. We suggest that strategies for reducing litter (fuel) accumulation and maintaining vigor of perennial bunchgrasses should be

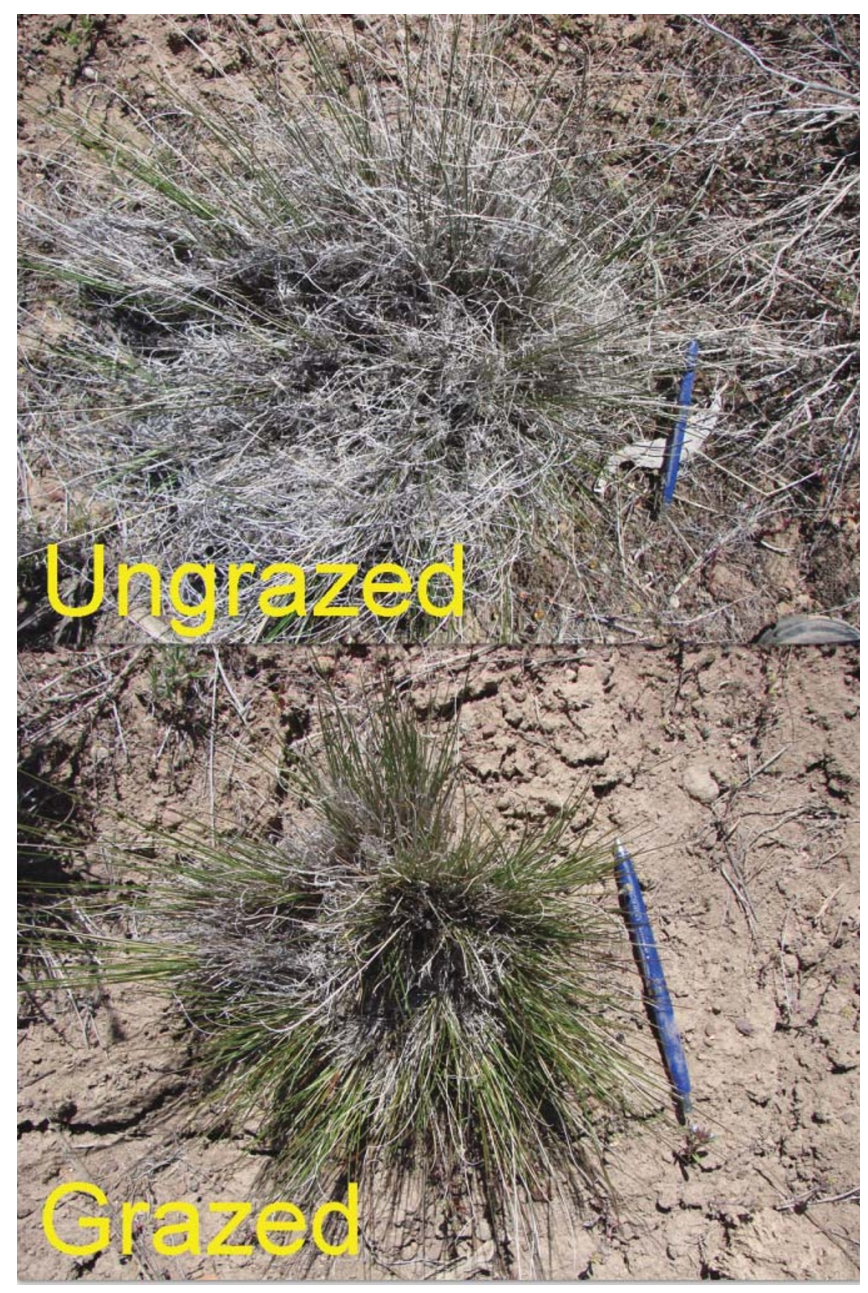

Figure 5. Photos taken on the same day showing long-term (70+ years) ungrazed (top) and moderately grazed (bottom) Thurber's needlegrass plants. Notice the accumulation of previous years' growth on the crown area of the ungrazed plant.

incorporated into management plans for sagebrush-steppe rangelands at risk of medusahead invasion. Likely, the only practical means of reducing litter accumulation of perennial bunchgrasses extensively on rangelands is through managed livestock grazing.

\section{Eradicating New Infestations of Medusahead}

Despite having the very best strategies in place for reducing the spread of medusahead seed and limiting resource availability to medusahead, uncontrollable events can still lead to new infestations. In addition, controlling new weed infestations is a more effective strategy than trying to control large infestations. ${ }^{18,19}$ We cannot overlook the importance of controlling incipient infestations of medusahead while they are still small and manageable. The smaller the infestation and the earlier it is detected, the greater the chance for successful eradication. The goal of eradicating new infestations of medusahead is to limit reproduction and subsequent development of a soil seed bank. Early detection of new 


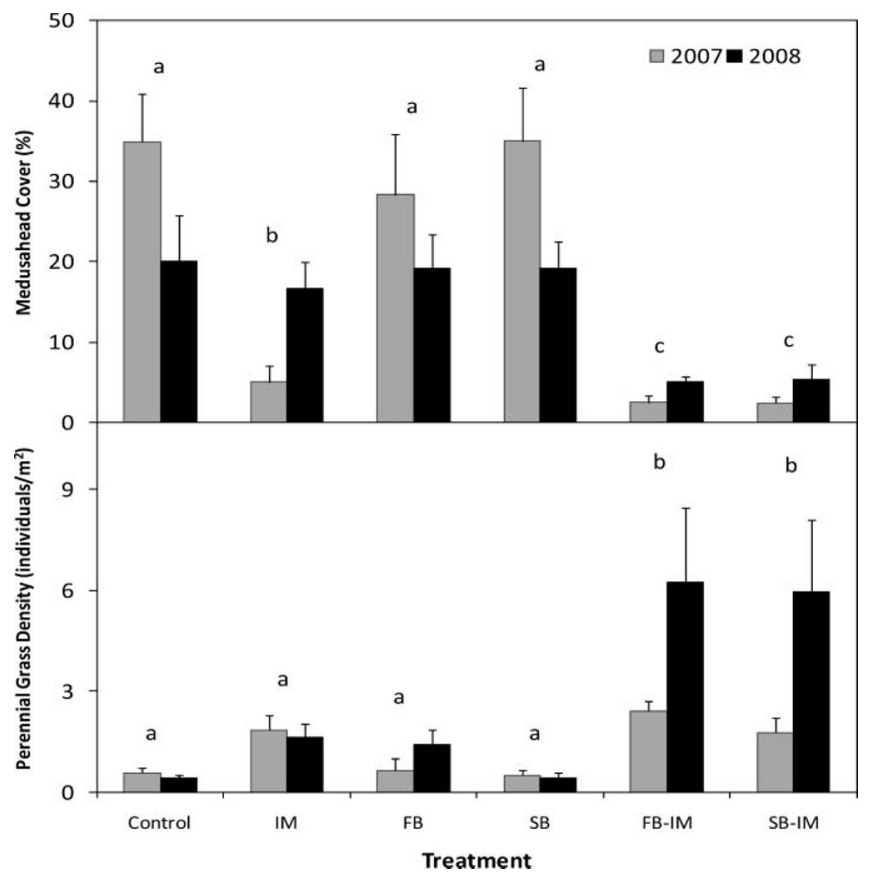

Figure 6. Medusahead cover (top mean \pm SE) and perennial bunchgrass density (bottom mean \pm SE) in 2007 and 2008, 1 and 2 years following various medusahead control treatments, respectively. Treatment key: Control = control, $\mathrm{IM}=$ fall imazapic application, $\mathrm{SB}=$ prescribed spring burn, $\mathrm{SB}-\mathrm{IM}=$ prescribed spring burn followed with fall imazapic application, $\mathrm{FB}=$ prescribed fall burn, $\mathrm{FB}-\mathrm{IM}=$ prescribed fall burn followed with fall imazapic application. Different lower case letters indicate differences between treatments after treatment applications $(P<0.05$; Adapted from Davies and Sheley' ${ }^{21}$.

infestations of medusahead is absolutely critical to successful eradication. Time and resource limitations, coupled with the vast nature of rangeland make searching all areas for new infestations untenable; this suggests that there is a critical need to employ a strategic approach for planned searches. One of the most logical ways to conduct systematic surveys for new infestations is to search around established medusahead populations and along major vector pathways. Because vehicles and animals are the primary dispersers of medusahead seed, searching for new infestations along roads, ATV trails, and livestock trails is a logical strategy. There is little substitute for vigilance, spending time out on the ground, and knowing which areas are at risk of invasion for improving our ability for locating and eradicating new infestations of medusahead.

\section{Options for Managing Existing Populations of Medusahead}

Once medusahead has developed a population that is too large and persistent to be targeted for eradication, control becomes the only management option, and the need for revegetation following control depends on the level of medusahead dominance. If perennial bunchgrasses are still present at acceptable densities, the objective should be to implement treatments that control medusahead while minimizing negative impacts to perennial bunchgrasses. The importance of retaining established perennial grasses cannot be overstated due to the expense and inherent challenges associated with revegetation of medusahead invaded rangeland. ${ }^{5,20}$ In a study of the effects of various combinations of medusahead control treatments in southeast Oregon, Davies and Sheley ${ }^{21}$ found that a fall- or spring-applied prescribed fire followed by an application of the pre-emergent herbicide, imazapic, at a rate of $87.5 \mathrm{~g}$ ai/ha in October provided the best medusahead control and fostered the greatest increase in perennial bunchgrass density (Fig. 6). Herbicide applications were made with a 10 -foot handheld $\mathrm{CO}_{2}$ sprayer (R \& D Sprayers, Opelousas, LA, USA) with a tank pressure of $206.8 \mathrm{kPa}$. Although an October application of imazapic was successfully used to suppress medusahead in the study in southeast Oregon, it is important to note that the appropriate application timing could be any time prior to germination of medusahead during late summer or fall. In addition, the area had accumulated a relatively thick layer of medusahead thatch, and burning served to reduce the litter layer and expose the soil surface for improved herbicideto-soil contact, thus affecting a greater pre-emergence control of medusahead. Burning removes the favorable conditions for germination and establishment of medusahead that are created when plant litter covers the soil surface. In a Nevada study, emergence of medusahead under litter was 47 times greater than emergence on bare ground by the end of March. ${ }^{22}$ By the end of the growing season, medusahead yield was times greater where persistent medusahead litter covered the soil than on bare soil. In addition to improving soil coverage and activity of imazapic, reducing the thatch layer and exposing the soil surface by burning likely contributed to the reduction in medusahead germination and subsequent production found in the Davies and Sheley ${ }^{21}$ trial. In some situations, the use of prescribed burning is precluded either by resource or liability concerns (particularly on private lands); thus, mechanical methods, such as disking, mowing, or raking, ${ }^{23}$ need to be considered for managing medusahead thatch prior to herbicide application. The ability to capitalize on the occurrence of wildfire with herbicide and seeding treatments (if needed) also is critical for areas where constraints to using prescribed burning cannot be overcome. A priori knowledge of where medusahead infestations are located and access to management resources are needed to take advantage of the conditions of a greatly reduced thatch layer that results from wildfire.

On many medusahead-invaded sites, perennial bunchgrasses are insufficient or even largely absent. In this case, revegetation is required and the strategy should be to control medusahead and promote seeded perennial bunchgrasses. The objective should be to provide seeded perennial bunchgrasses the largest window of opportunity possible for establishment in the absence of medusahead competition. This is most often accomplished with a prescribed burn followed by a fall-applied pre-emergence herbicide, such as imazapic. In 


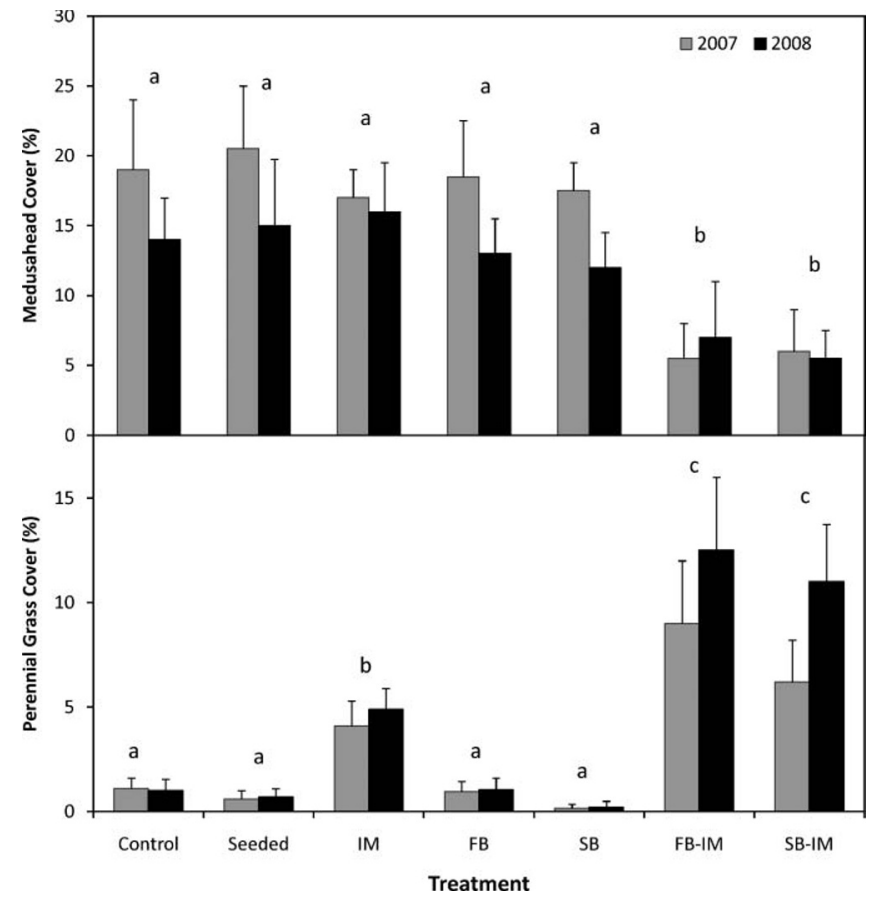

Figure 7. Medusahead cover (top mean \pm SE) and perennial bunchgrass cover (bottom mean \pm SE) in 2008 and 2009, 2 and 3 years following various medusahead control treatments, respectively. Treatment key: Control = control, Seeded = no medusahead control, IM= fall imazapic application, $\mathrm{SB}=$ prescribed spring burn, $\mathrm{SB}-\mathrm{IM}=$ prescribed spring burn followed with fall imazapic application, $\mathrm{FB}=$ prescribed fall burn, $\mathrm{FB}-\mathrm{IM}=$ prescribed fall burn followed with fall imazapic application. All plots but the control were drill seeded with desert wheatgrass and squirreltail at $15 \mathrm{~kg} / \mathrm{ha}$ (Adapted from Davies ${ }^{24}$ ).

situations where perennial bunchgrasses and other desirable vegetation are largely absent, spring treatments of preemergent herbicides combined with nonselective, postemergent herbicides, such as glyphosate, can be used. Such combinations not only deliver residual pre-emergent control of medusahead, but also have the added advantage of providing control of medusahead that has already germinated and emerged. Use of such herbicide combinations can also be applicable for situations that require concurrent control of perennial invasive plants and medusahead. The jury is still out on the efficacy of using a one pass system (i.e., herbicide application and seeding in one pass) with available pre-emergence herbicides. Our current recommendation is to wait one full growing season before performing a seeding of perennial bunchgrasses the subsequent fall to reduce risk of herbicide activity on seeded desirable vegetation. In an investigation of various medusahead control treatments combined with a dormant season seeding of perennial bunchgrasses, Davies ${ }^{24}$ concluded that a spring- or fall-applied prescribed burn followed by a fall application of imazapic at a rate of $87.5 \mathrm{~g}$ ai/ha provided the best medusahead control and fostered the greatest establishment of a seeding of perennial bunchgrasses (when seeded the fall after application of imazapic; Fig. 7).

\section{Conclusions}

Medusahead negatively effects biodiversity, grazing capacity of rangelands, and wildlife habitat of western rangelands. Medusahead, like other invasive annual grasses, also increases the size and frequency of wildfires, often to the detriment of desirable rangeland vegetation. Medusahead invasion is particularly troublesome to ranchers and other managers of western rangelands because areas invaded by the exotic annual grass have proven exceedingly difficult to revegetate and, without establishment of desirable plants, medusahead soon regains dominance of the plant community. However, recent research suggests that medusahead control and revegetation can be achieved with prescribed burning followed by a fall application of imazapic, and then a dormant seeding of desirable plant species the fall subsequent to herbicide treatment. It is important, however, to be mindful that the strategy requires three separate entries (i.e., prescribed burning, herbicide application, and seeding the fall subsequent to herbicide treatment) and is thereby expensive. In addition, most studies that support this conclusion have employed small treatment areas that might or might not be applicable to real-world management scenarios. There is a critical need to test these and other integrated control and revegetation strategies at a meaningful scale to provide land managers solid management recommendations for medusaheadinvaded rangeland.

A focus on prevention strategies was provided in this paper because we feel that limiting the spread of medusahead is a more effective use of weed management resources than focusing on control and restoration of existing infestations. Adopting an approach to managing medusahead that focuses on preventing new infestations and limiting expansion of existing infestations has the potential to preclude the need for revegetation on millions of acres. Adopting such an approach does not eliminate the need to restore alreadyinvaded rangeland plant communities, but suggests that with current resource and knowledge constraints, prevention is a more logical priority than restoration in most circumstances. Furthermore, the cumulative benefits of preventing the establishment of medusahead infestations are exponential because each infestation prevented eliminates all future descendant populations.

\section{Acknowledgments}

The authors appreciated the thoughtful reviews of the manuscript by Drs Brenda Smith and Jeremy James. The authors also thank the anonymous reviewers for their constructive comments.

\section{References}

1. Howell, T. 1903. A flora of northwest America. Vol. 1. Phanerogamae. Portland, OR, USA. Binford and Mort. 770 p.

2. Pellant, M., and C. Hall. 1994. Distribution of two exotic grasses on Intermountain rangelands: status in 1992. In: S. B. Monsen and S. G. Kitchen [comps.]. Proceedings: Ecology and 
Management of Annual Rangelands; 18-22 May 1992; Boise, ID, USA. Ogden, UT, USA: United States Department of Agriculture, Forest Service, Intermountain Research Station. General Technical Report INT-GTR-313. p. 109-112.

3. Young, K., And J. Mangold. 2008. Medusahead outperforms squirreltail through interference and growth rate. Invasive Plant Science and Management 1:73-81.

4. Hironaka, M. 1961. The relative rate of root development of cheatgrass and medusahead. Journal of Range Management 14:263-267.

5. Young, J. A. 1992. Ecology and management of medusahead (Taeniatherum caput-medusae ssp. asperum [Simk.] Melderis). The Great Basin Naturalist 52:245-252.

6. Torell, P. J., L. C. Erickson, and R.H. Haas. 1961. The medusahead problem in Idaho. Weeds 9:124-131.

7. Davies, K. W., and T. J. Svejcar. 2008. Comparison of medusahead-invaded and noninvaded Wyoming big sagebrush steppe in southeastern Oregon. Rangeland Ecology E' Management 61:623-629.

8. Miller, H. C., D. Clausnitzer, and M. M. Borman. 1999. Medusahead. In: R. L. Sheley and J. K. Petroff [EDs.]. Biology and management of noxious weeds. Corvallis, OR, USA: Oregon State University Press. p. 271-281.

9. Monaco, T. A., T. M. Osmond, and S. A. Dewey. 2005. Medusahead control with fall- and spring-applied herbicides on northern Utah foothills. Weed Technology 19:653-658.

10. Davies, K. W., and D. D. Johnson. 2008. Managing medusahead is at a critical threshold in the Intermountain West. Rangelands 30(4):13-15.

11. Davies, K. W. 2008. Medusahead dispersal and establishment in sagebrush steppe plant communities. Rangeland Ecology $\xi^{\circ}$ Management 61:110-115.

12. Davies, K. W., A. M. Nafus, and R. L. Sheley. 2010. Non-native competitive perennial grass impedes the spread of an invasive annual grass. Biological Invasions 12:3187-3194.

13. Davis, M. A., J. P. Grime, and K. Thomspon. 2000. Fluctuating resources in plant communities: a general theory of invisibility. Journal of Ecology 88:528-534.

14. James, J. J., K. W. Davies, R. L. Sheley, and Z. T. Aanderud. 2008. Linking nitrogen partitioning and species abundance to invasion resistance in the Great Basin. Oecologia 156:637-648.
15. Sheley, R. L., B. S. Bingham, and T. J. Svejcar. 2008. Crested wheatgrass defoliation intensity and season on medusahead invasion. Rangeland Ecology \& Management 61:211-217.

16. Claunnitzer, D. W., M. M. Borman, and D. E. Johnson. 1999. Competition between Elymus elymoides and Taeniatherum caput-medusae. Weed Science 47:720-728.

17. Davies, K. W., T. J. Svejcar, and J. D. Bates. 2009. Interaction of historical and nonhistorical disturbances maintains native plant communities. Ecological Applications 19:1536-1545.

18. Moody, M. E., ANd R. N. Mack. 1998. Controlling the spread of plant invasions: the importance of nascent foci. Journal of $A p-$ plied Ecology 25:1009-1021.

19. Smith, H. A., W. S. Johnson, J. S. Shonkwiler, and S. R. Swanson. 1999. The implications of variable or constant expansion rates in invasive weed infestations. Weed Science $47: 62-66$.

20. Young, J. A., C. D. Clements, and G. Nader. 1999. Medusahead and clay: the rarity of perennial seedling establishment. Rangelands 21(6):19-23.

21. Davies, K. W., and R. L. Sheley. 2011. Promoting native vegetation and diversity in exotic annual grass infestations. Restoration Ecology 19:159-165.

22. Evans, R. A., And J. A. Young. 1970. Plant litter and establishment of alien annual weed species in rangeland communities. Weed Science 18:697-703.

23. Kyser, G. B., J. M. DiTomaso, M. P. Doran, S. B. Orloff, R. B. Wilson, D. L. Lancaster, D. F. Lile, and M. L. Porath. 2007. Control of medusahead (Taeniatherum caputmedusae) and other annual grasses with imazapic. Weed Technology 21:66-75.

24. Davies, K. W. 2010. Revegetation of medusahead-invaded sagebrush steppe. Rangeland Ecology Eं Management 63:564-571.

Authors are Extension Agent, Dept of Rangeland Ecology and Management, Oregon State University Extension Service, Corvallis, OR 97331, USA, dustin.johnson@oregonstate.edu (Johnson); and Rangeland Scientist, USDA Agricultural Research Service, Eastern Oregon Agricultural Research Center, Burns, OR 97720, USA (Davies). 